\title{
Creencias de profesores chilenos de biología sobre la preparación de la enseñanza
}

\author{
Eduardo Ravanal Moreno ${ }^{1, a}$, Francisco López-Cortés ${ }^{2, b}$, Luis Rodríguez Moreno ${ }^{3, c}$ \\ ${ }^{1}$ Facultad de Pedagogía, Universidad Academia de Humanismo Cristiano.Santiago. Chile. \\ ${ }^{2}$ Departamento de Biología, Facultad de Ciencias, Universidad de La Serena. La Serena. Chile. \\ ${ }^{3}$ Facultad de Ciencias de la Educación, Universidad Metropolitana de Ciencias de la Educación. Chile. \\ areravanal@academia.cl,bflopez@userena.cl, clhrodriguezmo@gmail.com
}

[Recibido: 16 Noviembre 2017. Revisado: 26 Enero 2018. Aceptado: 26 Marzo 2018]

\begin{abstract}
Resumen: Las creencias y conocimientos del profesorado son asuntos importantes de explorar por la influencia que tienen sobre la preparación y enseñanza de la biología. Las creencias son las de mayor influencia en el quehacer profesional docente, y por esta razón nuestro interés en explorarlas. El estudio centra su atención en creencias del profesor de biología en servicio sobre la preparación de la enseñanza de la biología y su relación con algunas dimensiones de la evaluación docente en Chile. Para ello, se aplica un cuestionario con escala Likert para la preparación de la enseñanza de biología (PEB) y enseñanza de la biología (EB), que fue aplicado a 92 profesores de biología en ejercicio de la Región Metropolitana y Región de Coquimbo, Chile. En este artículo se presentan los resultados del análisis de la dimensión PEB. El análisis adopta un enfoque descriptivo-interpretativo complementado con el uso de técnicas cuantitativas. Los resultados señalan que el profesorado adscribe a todas aquellas creencias que representen una perspectiva constructivista de la preparación de la enseñanza; sin embargo, dicha tendencia cambia cuando el profesorado declara lo que cree hacer en la sala de clases, siendo las creencias de tendencia tradicional las más representativas. Evidenciamos algunas controversias entre lo que se piensa que se debería hacer y lo que se piensa que se hace, particularmente cuando se habla de la organización del contenido disciplinar y el sentido de la evaluación en biología. La exploración de las creencias y el desempeño del profesorado deben ayudarnos a pensar cómo orientar los procesos de formación inicial y continua que provean mejores resultados de desempeño y de aprendizaje para el profesorado y los estudiantes.
\end{abstract}

Palabras clave: Creencias; profesor de biología; preparación de la enseñanza; evaluación docente.

\section{Belief of Chilean biology teacher on the Preparation of Teaching}

Abstract: Teachers' beliefs and knowledge are important issues to explore because of the influence they have on the preparation and teaching of biology. Beliefs are the most influential aspects in the teaching profession, and for this reason our interest on exploring them. The study focuses on the in-service biology teachers' beliefs on the preparation of biology teaching and its relationship with some dimensions of the teaching assessment in Chile. To do that, a Likert-scale questionnaire for the preparation of biology teaching (PEB) and biology teaching (EB) was applied to 92 in-service biology teachers in the Metropolitan Region and Coquimbo Region, Chile. This article presents results of an analysis of the PEB dimension. The analysis adopts a descriptiveinterpretative approach complemented with the use of quantitative techniques. The results indicate that the teachers ascribe to all those beliefs that represent a constructivist perspective of the preparation of teaching; nevertheless, that tendency changes when the teachers declare what they believe to do in the classroom, being the beliefs of traditional tendency the most representative. We find some controversies between what the teachers think it should be done and what is thought to be done, particularly when it is referred to the matter subject organization and meaning of assessment in biology. The exploration the teachers' beliefs and performance should help us to think about how to guide the processes of initial and ongoing teacher training in order to improve the performance and learning outcomes for both teacher and students.

Keywords: beliefs, biology teacher, Preparation of Teaching, teaching assessment

Para citar este artículo: Ravanal E., López-Cortés F., Rodríguez L. (2018) Creencias de los profesores de biología sobre la Preparación de la Enseñanza. Revista Eureka sobre Enseñanza y Divulgación de las Ciencias 15(3), 3601. doi: 10.25267/Rev_Eureka_ensen_divulg_cienc.2018.v15.i3.3601 


\section{Introducción}

La escuela siempre ha promovido oportunidades de desarrollo personal y cognitivo para todos con el propósito de construir una sociedad más justa e igualitaria, que valore y respete a los otros y su diversidad (Mansour y Wegerif 2013). Para ello, el profesor y la enseñanza que se promueva es clave. Además, se reconoce que la enseñanza no se remite solo al aprendizaje de conocimientos para un tipo de evaluación, sino también a favorecer las maneras de pensar para que todos participen con éxito del mundo cotidiano. La escuela y las clases de ciencias que en ella ocurren, son las responsables de promover un pensar teórico (Ravanal y Quintanilla 2012) que desarrolle y amplíe en los estudiantes la comprensión de los fenómenos del mundo natural, así como la capacidad de actuar y decidir considerando sus principios morales y éticos, si son necesarios. La escuela es un lugar para el diálogo, el debate y los cuestionamientos, definiéndose como el espacio para reestructurar las ideas de y sobre ciencia. Por ello, el profesor debe migrar hacia modelos teóricos coherentes con esa conceptualización de la enseñanza de la ciencia -biología- y responder a las demandas socio-científicas (Quintanilla 2006) de un país en desarrollo, que enfrenta con optimismo los desafíos del siglo XXI.

Modelos de enseñanza que no reconozcan los atributos y las demandas de una enseñanza centrada en el estudiante, restringen las intenciones de una formación integral, constituyéndose en un desafío para la formación de profesores y de cualquier programa de desarrollo profesional que pretenda contribuir a una nueva práctica de enseñanza. Sin embrago, la investigación sigue reportando que el modelo tradicional es el dominante en ciencias (Pujalte, Adúriz-Bravo y Porro 2015), siendo las creencias ingenuas las orientadoras de ese tipo de enseñanza (Guerra y Sabastián 2015). Las creencias, en ese sentido, actúan como filtros y esquemas de interpretación de la información, por ende, guían la planificación y las acciones de enseñanza (Fives y Buehl 2012). Éstas son un tipo de representación mental que influye en las acciones del profesor (Hutner y Markman 2016) y en los resultados de aprendizaje (Soini, Pietarinen \& Pyhältö 2016), por eso el interés de explorarlas.

El objetivo del presente estudio es explorar las creencias de profesorado chileno de biología, en servicio, sobre la preparación de la enseñanza de la biología (PEB) y la relación de estas con las dimensiones de la evaluación docente en Chile.

\section{Marco teórico}

Las creencias y experiencias del profesor influyen en la comprensión que se tiene de la enseñanza y, por tanto, en la representación profesional sobre las demandas y exigencias de preparación e implementación de la enseñanza. Dicha comprensión orienta las decisiones del qué y cómo preparar la enseñanza, así como del qué y cómo implementarla en pos de los aprendizajes (Kind 2015). La demanda profesional implícita en lo anterior, indiscutiblemente, representa una gama de actividades sustentadas en el conocimiento, las creencias y las experiencias del profesor, siendo las creencias, las principales predictoras en dichas actividades (Pajares 1992) y, por tanto, las de mayor influencia sobre lo planificado -deseado- e implementado -realizado- en el aula.

Muchas de las acciones profesionales del profesor -como son planificar, enseñar y evaluarestán condicionadas por el sistema de creencias y conocimientos que los profesores han construidos en su quehacer (Hoffman y Seidel 2015) y sobre las cuales las creencias constituyen los filtros cognitivos más influyentes, especialmente sobre qué y cómo aprenden las personas (Wallace y Loughran 2012). En el sistema de creencias del profesor están las llamadas creencias epistemológicas (Bahçivan 2016 ; Hofer y Pintrich 1997; Wallace 2015 ), 
reconocidas como estructuras cognitivas individuales acerca del conocimiento y del aprendizaje (Guerra y Sebastián 2015), que por cierto, afectan la conceptualización de la preparación de la enseñanza y la forma de trasmitirla (Kang 2008), de ahí la importancia de ampliar la comprensión sobre ellas e identificar todas aquellas que pudieran afectar positiva o negativamente la enseñanza para el aprendizaje. Una mayor y mejor comprensión de lo que representa una creencia en el profesor, constituye una oportunidad de aprendizaje para él, en la medida que reconozca el valor predictivo inicial y su estatus de esquema de pensamiento de influencia superior al conocimiento académico (Bryan 2012).

Desde la perspectiva cognitiva, la preparación de la enseñanza (PE) implica para el profesor explicitar su conocimiento tácito (Batatia, Hakkarainen y Morch 2012), resultado de la experiencia individual y de sus creencias (Grangeat y Kapelari 2015) y reconocer que los estudiantes aprenden en un espacio y tiempo determinado según su naturaleza personal. Durante la preparación de la enseñanza, el profesor no solo externaliza su conocimiento tácito, sino también, la visión del conocimiento a enseñar; absoluto, tentativo o evolutivo. Reconoce implícitamente, su nivel de conocimiento sobre lo que debe o desea enseñar, en ese contexto, las creencias y el conocimiento operan en conjunto como un tipo de representación mediadora (Hutner y Markman 2016) para filtrar la información y delimitar operativamente la preparación de la enseñanza (Fives y Buehl 2012; Buehl y Beck 2015) reconociendo en ello que las creencias constitutivas de dicha representación mediadora no actúan una independiente de la otra, sino como un sistema que distinguen creencias centrales y periféricas (Bryan 2012, Contreras 2010) o creencias ingenuas -enseñanza tradicional- y creencias sofisticadas -enseñanza constructivista- (Lee y Tsai 2011).

En Chile, existe un instrumento regulador para la promoción de una enseñanza de calidad, conocido como Marco para la Buena Enseñanza (Ministerio de Educación [MINEDUC], 2008). En él se señala que el proceso de enseñanza exige al profesor un conocimiento específico que permita organizarla para lograr aprendizajes en sus estudiantes. En ese contexto, la preparación y organización de la enseñanza implican al menos, dominar el contenido de la disciplina -la materia- que se enseña, como los aspectos que favorecen su aprendizaje. Dominar la materia que se enseña obliga saber distinguir los conceptos centrales y periféricos que la articulan, como la relación que existe entre ellos y con los métodos y procedimientos que la validan como un nuevo conocimiento (Grossman, Wilson y Shulman 2005). En ese plano, la comprensión de la materia resulta ser un desafío si la pretensión es una enseñanza eficaz (Scheerens 2016). Para Kennedy (1998), la materia es comprendida por el profesor, cuando este distingue y relaciona las ideas centrales y fundamentales de las periféricas o minucias. Así, las creencias que se tenga sobre el conocimiento y comprensión de la materia, ya sea ingenuas o sofisticadas determinarían las decisiones del qué enseñar y de cómo organizarlas para ello, como del qué evaluar y cómo hacerlo para el aprendizaje por su incidencia en el conocimiento didáctico del profesor (Park, Suh y Seo 2017), de ahí la importancia de explorarlas para mejorar su comprensión.

\section{Metodología}

En el marco del proyecto Fondecyt 11140297 se diseña un estudio exploratorio transeccional de las creencias del profesorado de biología sobre la preparación de la enseñanza de la Biología. Las variables de interés para el estudio y su operacionalización se muestran en la Tabla 1. 
Tabla 1. Variables en estudio organizadas por dimensión, plano de pensamiento y visión epistemológica.

\begin{tabular}{|c|c|c|c|}
\hline $\begin{array}{c}\text { Dimensión a } \\
\text { estudiar }\end{array}$ & $\begin{array}{c}\text { Plano del } \\
\text { pensamiento }\end{array}$ & $\begin{array}{c}\text { Visión } \\
\text { epistemológica }\end{array}$ & Operacionalización del plano de pensamiento \\
\hline \multirow{4}{*}{$\begin{array}{l}\text { Preparación de } \\
\text { la Enseñanza } \\
\text { de la Biología } \\
\text { (PEB) }\end{array}$} & \multirow[t]{2}{*}{ Pensamiento } & Tradicional (T) & \multirow{2}{*}{$\begin{array}{l}\text { Ideas que expresen lo que se cree debería hacer el } \\
\text { profesor cuando Prepara la Enseñanza de la Biología } \\
\text { según una perspectiva de acceso al conocimiento ( } \mathrm{T} \text { o C). }\end{array}$} \\
\hline & & Constructivista $(\mathrm{C})$ & \\
\hline & \multirow[t]{2}{*}{ Acción } & Tradicional (T) & \multirow{2}{*}{$\begin{array}{l}\text { Ideas que expresen lo que cree hacer el profesor cuando } \\
\text { Prepara la Enseñanza de la Biología según una perspectiva } \\
\text { de acceso al conocimiento ( } \mathrm{T} \text { o } \mathrm{C}) \text {. }\end{array}$} \\
\hline & & Constructivista (C) & \\
\hline
\end{tabular}

\section{Descripción de los participantes}

Participan 92 profesores de biología en servicio quienes fueron contactados por correo electrónico para participar de un seminario sobre Desarrollo Profesional Docente. La actividad convocante se realizó en la Región Metropolitana y en la de Coquimbo, Chile. La información se obtuvo desde bases de datos de los centros de prácticas disponibles para cada región. Los datos sociodemográficos de la muestra se indican en la Tabla 2.

Tabla 2. Antecedentes sociodemográficos de los profesores de biología participantes.

\begin{tabular}{|c|c|c|c|c|c|c|}
\hline Antecedentes & \multicolumn{6}{|c|}{ Plano Distribución (\%) } \\
\hline Rango de edad & \multicolumn{2}{|c|}{ De 25 a 35 años $(46,5 \%)$} & \multirow{2}{*}{\multicolumn{2}{|c|}{$\begin{array}{c}\text { De } 36 \text { a } 45 \text { años } \\
(33,7 \%)\end{array}$}} & \multirow{2}{*}{\multicolumn{2}{|c|}{$\begin{array}{c}\text { De } 46 \text { o más años } \\
(19,8 \%) \\
\text { De } 26 \text { o más años } \\
(9,4 \%)\end{array}$}} \\
\hline $\begin{array}{l}\text { Rango de años de } \\
\text { experiencia docente }\end{array}$ & $\begin{array}{c}\text { De } 0 \text { a } 5 \text { años } \\
(36,5 \%)\end{array}$ & $\begin{array}{l}\text { De } 6 \text { a } 15 \text { años } \\
\quad(40,0 \%)\end{array}$ & & & & \\
\hline $\begin{array}{l}\text { Género de los } \\
\text { participantes }\end{array}$ & \multicolumn{3}{|c|}{$\begin{array}{l}\text { Hombre } \\
(25,3 \%)\end{array}$} & \multicolumn{3}{|c|}{$\begin{array}{l}\text { Mujer } \\
(74,7 \%)\end{array}$} \\
\hline $\begin{array}{l}\text { Niveles educativos que } \\
\text { atiende }\end{array}$ & $\begin{array}{c}\text { Primaria } \\
\text { completa } \\
(1,2 \%)\end{array}$ & $\begin{array}{c}\text { Primaria parcial } \\
\qquad(4,7 \%)\end{array}$ & $\begin{array}{c}\text { Primaria y } \\
\text { Secundaria } \\
\text { parcial } \\
(8,2 \%)\end{array}$ & $\begin{array}{c}\text { Primaria y } \\
\text { Secundaria } \\
\text { completa } \\
(10,6 \%)\end{array}$ & $\begin{array}{c}\text { Secundaria } \\
\text { completa } \\
(30,6 \%)\end{array}$ & $\begin{array}{c}\text { Secundaria } \\
\text { parcial } \\
(44,7 \%)\end{array}$ \\
\hline
\end{tabular}

\section{Instrumento y procedimiento de recogida de datos}

La recogida de información se realizó mediante el cuestionario de Pensamiento y Acción sobre la preparación y enseñanza de la Biología, el cual se organizó en tres secciones: Antecedentes demográficos del profesor (sección 1) y las creencias de los participantes sobre la Dimensión de Enseñanza de la biología (EB) y su Preparación (PEB), esta última discutida en este artículo. En un plano declarativo, el cuestionario explora lo que el profesor piensa que debería hacer cuando prepara y enseña biología (sección 2 - denominada "Pensamiento") y lo que piensa que hace cuando prepara y enseña biología (sección 3 - denominada "Acción"). El cuestionario fue diseñado y validado en torno a dos dimensiones: PEB y EB, las que a su vez, representan dos planos del pensamiento del profesor: Pensamiento y Acción según una visión epistemológica particular, tradicional (T) o constructivista (C) según Contreras (2010) sobre Pensamiento y Actuación educativa para profesores chilenos. Los enunciados del cuestionario sobre planificación que hemos analizado se muestran en las tablas 3, 4 y 5, con excepción de los enunciados 2 y 3 que aluden a la enseñanza de la biología.

Para la validez de contenido, se utilizó la fórmula de Lawshed (1975) y el ajuste de TristánLópez (2008) para 5 jueces, que establece que 0,3 es el valor mínimo de razón de validez para cada enunciado. Enunciados que no cumplieran con la exigencia fueron eliminados. 
Posteriormente se evaluó la confiablidad del instrumento a través del Alpha de Cronbach, obteniendo un valor de 0,744 para la dimensión PEB. El cuestionario definitivo quedó conformado por 31 enunciados -ítems-, de los cuales 15 representan la Dimensión PEB y 16 ítems la Dimensión EB. Este artículo solo analiza PEB.

Cada enunciado de la sección "Pensamiento" tiene 4 opciones de respuesta con escala de valoración tipo Likert, que van desde Totalmente de acuerdo (TA), Parcialmente de acuerdo (PA), En desacuerdo (ED) a Totalmente en desacuerdo (TD). En tanto, la sección "Acción" utiliza 4 opciones que van desde Siempre (S), Frecuentemente (FQ), Rara vez (R) y Nunca (N). La puntuación para la escala nominal usada depende de la visión epistemológica que represente cada enunciado. Los enunciados usados en la sección "Pensamiento" son los mismos que se usan en la sección "Acción", solo cambia su redacción ya que estos últimos están expresados en la forma de procedimientos. Por ejemplo, el enunciado E1 plantea: "Los contenidos de biología se deben organizar de tal forma que se relacionen unos contenidos con otros", mientras que en la forma de "Acción" el planteamiento es: "Los contenidos de biología los organizo de tal forma que se relacionen unos contenidos con otros". Esto entrega antecedentes de ambos planos declarativos para un mismo enunciado.

\section{Técnicas de análisis de datos}

El análisis de los datos fue descriptivo, inferencial y multivariado. En términos descriptivos y para conocer el grado de adhesión a los enunciados se consideró el porcentaje obtenido por la suma de Totalmente de Acuerdo y Parcialmente de Acuerdo (TA+PA) para el plano del "Pensamiento" y la suma de las opciones Siempre y Frecuentemente (S+FQ) para el plano de la "Acción". Se utilizó el test de Wilcoxon para datos numéricos de una escala ordinal (Berlanga y Rubio 2012) para detectar diferencias significativas entre lo que se cree se debería hacer y lo que se cree se hace para cada enunciado. En adición, para conocer los factores que explican la adhesión de lo que creen los profesores respecto a $\mathrm{PEB}$, se realizó un análisis factorial. Finalmente y con el objetivo de establecer una relación entre la creencia declarada y la evaluación docente en Chile, se realizaron análisis de contenido global de cada enunciado para determinar su vínculo con tres dimensiones de la evaluación docente chilena (Tabla 3, 4 y 5). Para ello, se realiza un análisis de concordancia inter-observador entre los enunciados del cuestionario y los indicadores que definen las dimensiones de la evaluación docente de interés para el artículo. El análisis de concordancia arrojó un índice de kappa igual a 0,75 considerado bueno (Torres y Perera 2009). Los enunciados del cuestionario y la relación con los indicadores según dimensión de la evaluación docente se muestran en las tablas 3, 4 y 5 . 
Tabla 3. Relación de los enunciados sobre preparación de la enseñanza de la biología desde el plano del "Pensamiento" y "Acción" y la dimensión Organización de los Elementos de la Unidad de la evaluación docente chilena.

\begin{tabular}{|c|c|c|c|}
\hline $\begin{array}{l}\text { Enunciado } \\
\text { (E) y } \\
\text { Epistemología } \\
(\mathrm{T} / \mathrm{C})\end{array}$ & $\begin{array}{l}\text { Descripción del Enunciado del } \\
\text { cuestionario }\end{array}$ & $\begin{array}{l}\text { Indicador de } \\
\text { Evaluación Docente } \\
\text { y enunciados del } \\
\text { cuestionario }\end{array}$ & $\begin{array}{c}\text { Descripción operacional de la } \\
\text { dimensión Organización de los } \\
\text { Elementos de la Unidad } \\
(\mathrm{OEU})\end{array}$ \\
\hline E1C & $\begin{array}{l}\text { Los contenidos de biología [se deben } \\
\text { organizar/los organizo] de tal forma } \\
\text { que se relacionen unos contenidos } \\
\text { con otros. }\end{array}$ & \multirow{4}{*}{$\begin{array}{l}\text { Secuencia la clase para } \\
\text { el logro de objetivos. }\end{array}$} & \multirow{8}{*}{$\begin{array}{l}\text { Esta dimensión evalúa la } \\
\text { capacidad de organizar la clase } \\
\text { en una secuencia pedagógica y } \\
\text { didáctica que contribuya al } \\
\text { logro de los objetivos de } \\
\text { aprendizaje en los estudiantes. }\end{array}$} \\
\hline E3T & $\begin{array}{l}\text { [Lo apropiado es planificar/Planifico] } \\
\text { las actividades de los estudiantes para } \\
\text { los momentos de inicio, desarrollo y } \\
\text { cierre de clase. }\end{array}$ & & \\
\hline E4T & $\begin{array}{l}\text { [Lo más adecuado es } \\
\text { organizar/Organizo] los contenidos } \\
\text { de biología en una secuencia lógica y } \\
\text { lineal de temas. }\end{array}$ & & \\
\hline E31T & $\begin{array}{l}\text { Lo adecuado es organizar/Organizo] } \\
\text { los contenidos de biología en una } \\
\text { secuencia lineal que se ajusta a la } \\
\text { lógica de la disciplina que enseño. }\end{array}$ & & \\
\hline $\mathrm{E} 8 \mathrm{~T}$ & $\begin{array}{l}\text { Lo adecuado es planificar/Planifico] } \\
\text { las actividades de los estudiantes } \\
\text { coherentes con los objetivos de } \\
\text { aprendizaje definidos por el marco } \\
\text { curricular. }\end{array}$ & \multirow[t]{2}{*}{$\begin{array}{lr}\text { Capacidad de } & \text { generar } \\
\text { actividades } & \text { de } \\
\text { aprendizaje } & \text { que } \\
\text { contribuyan al } & \text { logro } \\
\text { de los objetivos. } & \end{array}$} & \\
\hline E11C & $\begin{array}{l}\text { [Es adecuado planificar/Planifico] una } \\
\text { secuencia de actividades que permita } \\
\text { socializar los objetivos de aprendizaje } \\
\text { de una clase de biología }\end{array}$ & & \\
\hline E12C & $\begin{array}{l}\text { Es apropiado Diseñar/Diseño] } \\
\text { estrategias didácticas que permita } \\
\text { abordar las dificultades que presentan } \\
\text { los estudiantes durante su aprendizaje. }\end{array}$ & \multirow{2}{*}{$\begin{array}{lrrr}\text { Capacidad } & & \text { para } \\
\text { reformular } & \text { estrategias } \\
\text { didácticas } & \text { según } & \text { los } \\
\text { resultados } & & \text { de } \\
\text { aprendizaje } & \text { de } & \text { los } \\
\text { estudiantes. } & & \\
& & \\
& & \\
& & \end{array}$} & \\
\hline E16C & $\begin{array}{l}\text { [Es adecuado preparar/Preparo] } \\
\text { diversas actividades de enseñanza para } \\
\text { que los estudiantes con distintas } \\
\text { capacidades logren los objetivos de } \\
\text { aprendizaje previstos. }\end{array}$ & & \\
\hline
\end{tabular}


Tabla 4. Relación de los enunciados sobre Preparación de la enseñanza de la biología desde el plano del "Pensamiento” y “Acción” y el indicador Reflexión Pedagógica para la evaluación de desempeño docente.

\begin{tabular}{|c|c|c|c|}
\hline $\begin{array}{c}\text { Enunciado } \\
\text { (E) y } \\
\text { Epistemología } \\
(\mathrm{T} / \mathrm{C})\end{array}$ & $\begin{array}{l}\text { Descripción del Enunciado del } \\
\text { cuestionario }\end{array}$ & $\begin{array}{l}\text { Indicador de } \\
\text { Evaluación Docente } \\
\text { y enunciados del } \\
\text { cuestionario }\end{array}$ & $\begin{array}{c}\text { Descripción operacional de la } \\
\text { dimensión Reflexión } \\
\text { Pedagógica }(\mathrm{RP})\end{array}$ \\
\hline E5T & $\begin{array}{l}\text { [Lo adecuado es planificar/Planifico] } \\
\text { la clase considerando que el } \\
\text { conocimiento de biología es producto } \\
\text { de la acumulación de teorías que han } \\
\text { sido comprobadas. }\end{array}$ & \multirow{3}{*}{$\begin{array}{l}\text { Analiza las decisiones } \\
\text { pedagógicas-didácticas } \\
\text { del contexto de } \\
\text { implementación de la } \\
\text { unidad y los objetivos } \\
\text { de aprendizaje. }\end{array}$} & \multirow{5}{*}{$\begin{array}{l}\text { Esta dimensión evalúa la } \\
\text { capacidad del profesor para } \\
\text { reflexionar sobre su práctica, } \\
\text { ya sea sobre su capacidad para } \\
\text { organizar el contenido según } \\
\text { su progresión y su reflexión a } \\
\text { partir de resultados de } \\
\text { enseñanza y aprendizaje. }\end{array}$} \\
\hline E6C & $\begin{array}{l}\text { Las estrategias de evaluación [deberían } \\
\text { ser elaboradas/las elaboro] con el } \\
\text { grupo de profesores de biología o de } \\
\text { ciencias de mi colegio. }\end{array}$ & & \\
\hline E39T & $\begin{array}{l}\text { [Se debe considerar en la } \\
\text { planificación/Planifico] la clase de } \\
\text { biología según los conocimientos } \\
\text { genéricos al que deben llegar los } \\
\text { estudiantes para demostrar que han } \\
\text { aprendido. }\end{array}$ & & \\
\hline E13C & $\begin{array}{l}\text { [Es adecuado evaluar/Evalúo] las } \\
\text { fortalezas y debilidades de una unidad } \\
\text { didáctica antes de implementarla en la } \\
\text { sala de clase. }\end{array}$ & \multirow{2}{*}{ 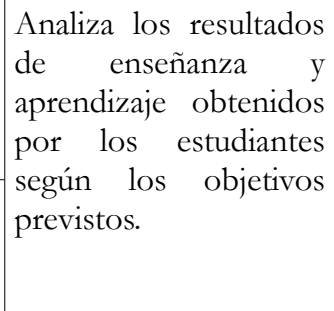 } & \\
\hline E15C & $\begin{array}{l}\text { [Es adecuado analizar/Analizo] los } \\
\text { resultados de las evaluaciones para } \\
\text { planificar y ajustar mi enseñanza. }\end{array}$ & & \\
\hline
\end{tabular}

Tabla 5. Relación de los enunciados sobre Preparación de la enseñanza de la biología desde el plano del "Pensamiento" y "Acción” y el indicador Evaluación de los Aprendizajes de la EDD.

\begin{tabular}{|c|c|c|c|}
\hline $\begin{array}{l}\text { Enunciado } \\
\text { (E) y } \\
\text { Epistemología } \\
(\mathrm{T} / \mathrm{C})\end{array}$ & $\begin{array}{l}\text { Descripción del Enunciado del } \\
\text { cuestionario }\end{array}$ & $\begin{array}{l}\text { Indicador de } \\
\text { Evaluación Docente } \\
\text { y enunciados del } \\
\text { cuestionario }\end{array}$ & $\begin{array}{c}\text { Descripción operacional de la } \\
\text { dimensión Evaluación de los } \\
\text { Aprendizajes (EV) }\end{array}$ \\
\hline E23T & $\begin{array}{l}\text { Para mantener el interés de los } \\
\text { estudiantes en la asignatura de } \\
\text { biología [es recomendable/defino] } \\
\text { evaluaciones sumativas frecuentes. }\end{array}$ & \multirow{2}{*}{$\begin{array}{l}\text { Evalúa para regular el } \\
\text { aprendizaje desde una } \\
\text { perspectiva sumativa y } \\
\text { formativa. }\end{array}$} & \multirow{2}{*}{$\begin{array}{l}\text { Esta dimensión evalúa la } \\
\text { capacidad de orientar a los } \\
\text { estudiantes en su aprendizaje a } \\
\text { partir del análisis los resultados } \\
\text { de aprendizaje. }\end{array}$} \\
\hline E25T & $\begin{array}{l}\text { [Lo fundamental es } \\
\text { planificar/Planifico] evaluaciones que } \\
\text { midan el aprendizaje de conceptos } \\
\text { biológicos. }\end{array}$ & & \\
\hline
\end{tabular}




\section{Resultados}

\section{a) Creencias del profesor de biología sobre la Preparación de la Enseñanza de la Biología}

El análisis estadístico descriptivo consideró el uso de la media aritmética y la desviación estándar, las que son complementadas con un análisis porcentual para cada uno de los enunciados (Tabla 6). Al respecto, podemos decir que la mayor y principal adhesión se da para enunciados de tipo constructivista, reconociendo en ello, que muchas de las creencias que se piensan para preparar la enseñanza de la biología, también se cree hacer en la sala de clases, exceptuado lo referido a la evaluación del aprendizaje (E6C).

Tabla 6. Grado de adhesión promedio ( $\pm 1 \mathrm{DE})$ y porcentual de $(\mathrm{TA}+\mathrm{PA})$ o $(\mathrm{S}+\mathrm{FQ})$ de los planos de "Pensamiento" y "Acción".

\begin{tabular}{|c|c|c|c|c|c|c|}
\hline \multicolumn{7}{|c|}{ Preparación de la Enseñanza de la Biología } \\
\hline \multirow[b]{2}{*}{ Enunciado } & \multicolumn{3}{|c|}{ Pensamiento } & \multicolumn{3}{|c|}{ Acción } \\
\hline & Media & $\mathrm{DE}$ & $\%(\mathrm{TA}+\mathrm{PA})$ & Media & $\mathrm{DE}$ & $\%(\mathrm{~S}+\mathrm{FQ})$ \\
\hline $3 \mathrm{~T}$ & 1,38 & 0,63 & 95,2 & 1,45 & 0,76 & 91,6 \\
\hline $4 \mathrm{~T}$ & 1,58 & 0,76 & 89,2 & 1,49 & 0,7 & 96,4 \\
\hline $5 \mathrm{~T}$ & 1,92 & 0,77 & 78,3 & 1,93 & 0,9 & 74,7 \\
\hline $8 \mathrm{~T}$ & 1,43 & 0,87 & 90,4 & 1,29 & 0,64 & 98,8 \\
\hline $23 \mathrm{~T}$ & 3,52 & 0,93 & 57,8 & 1,9 & 1,09 & 63,8 \\
\hline $25 \mathrm{~T}$ & 2,4 & 0,91 & 45,8 & 1,77 & 0,95 & 79,5 \\
\hline $31 \mathrm{~T}$ & 1,7 & 0,77 & 85,5 & 1,63 & 0,85 & 89 \\
\hline 39T & 1,63 & 0,47 & 91,6 & 1,68 & 0,81 & 88 \\
\hline $1 \mathrm{C}$ & 3,73 & 0,77 & 100 & 3,12 & 1,15 & 94 \\
\hline $6 \mathrm{C}$ & 3,4 & 0,88 & 92,8 & 2,05 & 1,21 & 39,8 \\
\hline $11 \mathrm{C}$ & 3,46 & 0,83 & 97,6 & 2,92 & 1,22 & 84,1 \\
\hline $12 \mathrm{C}$ & 3,63 & 0,81 & 98,8 & 3,07 & 1,13 & 91,6 \\
\hline $13 C$ & 3,36 & 0,83 & 97,6 & 2,78 & 1,13 & 80,7 \\
\hline $15 C$ & 3,7 & 0,79 & 98,8 & 3,04 & 1,15 & 88 \\
\hline $16 \mathrm{C}$ & 3,62 & 0,84 & 96,4 & 2,87 & 1,18 & 78,3 \\
\hline
\end{tabular}

Con el propósito de identificar las creencias del profesor sobre la PEB y ampliar el carácter exploratorio del estudio se realiza un análisis factorial (Figura $1 \mathrm{a}, \mathrm{b}$ ), que permitió distinguir dos grupos de enunciados, los de tendencia tradicional y los de tendencia constructivista. Independiente del plano con que fueron analizados ambos grupos comparten dentro de ellos elementos comunes referidos a: OEU, EA y EP (Tabla 3). El análisis factorial del plano del pensamiento -lo que se cree se debería hacer- permitió reconocer que las dos primeras componentes explican el $61,1 \%$ de la información de los datos, en donde el componente 1 está conformado por enunciados constructivistas -E12C, E15C, E16C, E1C, E11C, E13Csiendo la tendencia que mayormente explica la adhesión del profesorado a las creencias 
expresadas en el cuestionario (varianza explicada de 44,5\%) las que a su vez, se relacionan con la dimensión de Organización de Elementos de la Unidad (OEU) y de Reflexión Pedagógica (RP) de la evaluación de desempeño docente, El componente 2 está conformado por enunciados de tendencia tradicional -E4T, E31T, E39T, E5T, E8T, E3T- explicando el 16,5\% de la varianza total. El diseño de actividades (E16C) para lograr objetivos según las diferencias individuales (E16C) y el ajuste de la planificación y enseñanza derivados del análisis de los resultados de aprendizaje (E15C), son asuntos que representan las creencias del primer grupo, mientras que, el segundo grupo de enunciados está representado por creencias de tendencia tradicional relacionadas también, a las dimensiones OEU y RP, en la que organizar los contenidos de biología en una secuencia lógica y lineal para los momentos de inicio, desarrollo y cierre (E4T; E31T; E3T), como planificar la clase considerando que el conocimiento es producto de la acumulación de teorías (E5T) resultan ser las creencias más representativas, En tanto, los enunciados sobre la evaluación del aprendizaje (E25T; E23T) están menos correlacionados con dicha visión.
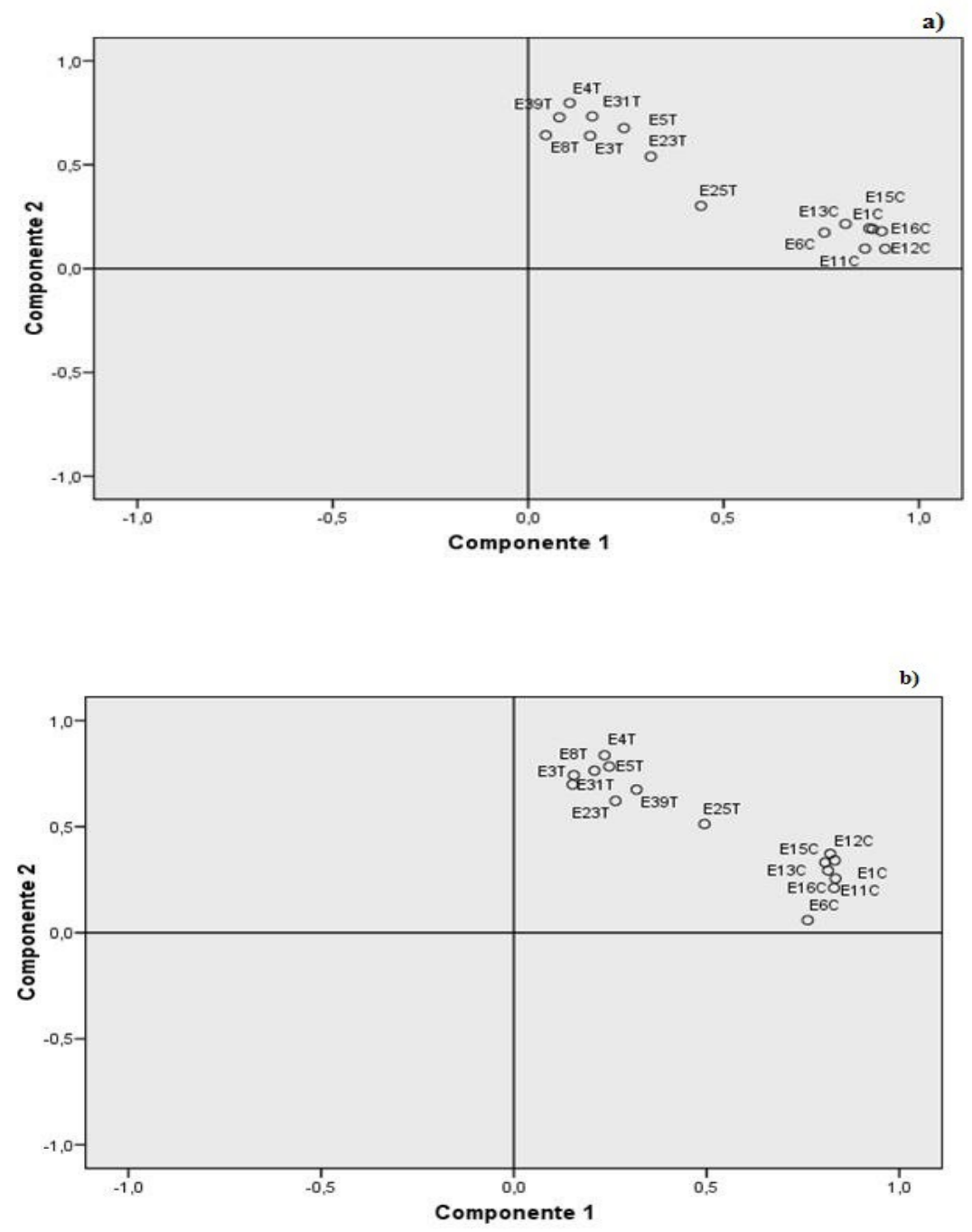

Figura 1. Análisis factorial del plano de a) Pensamiento $(\mathrm{KMO}=0,88)$ y b) Acción $(\mathrm{KMO}=0,91)$

El análisis factorial, desde el plano de la -Acción-, explica el 68,1\% del total de la información recogida. Al respecto, el componente 1 está conformado por enunciados constructivistas -E11C, E1C, E16C, E12C y E13C- y es el factor que mayormente explica la adhesión de los 
profesores a dichas creencias $(56.8 \%$ de la varianza total). Las creencias de tendencia constructivista vinculadas a OEU son las que más explican el conjunto de datos. En este sentido, la pretensión de lograr objetivos de aprendizajes (E11C), secuenciar el contenido relacionándolo uno con otro (E1C) y reformular las estrategias para lograr los objetivos según los estudiantes (E16C y E12C) constituyen el eje central para el profesor cuando prepara la enseñanza de la biología.

La prueba no paramétrica de Wilcoxon permitió establecer diferencias significativas para los siguientes pares de enunciados: E1C, E6C, E11C, E12C, E13C, E15C, E16C, E23T, E25T, revelando "rupturas" por plantearlo de alguna forma, entre lo que se cree se debe hacer y lo que se cree hacer cuando se planifica la enseñanza de la biología (Figura 2), observado mayoritariamente en enunciados de tendencia constructivista (C) y, particularmente en aquellos ligados a la organización del contenido disciplinar (E1), los objetivos, las estrategias y actividades de aprendizaje (E11, E12), el análisis de los resultados para la toma de decisiones (E13, E15) y la elaboración colaborativa de las estrategias de evaluación (E6). La PEB debe permitir vincular un contenido con otro, como hacer visible las razones que hay detrás de su aprendizaje, sin embargo, eso que parece trivial, se cree no hacer con frecuencia (Tabla 1).

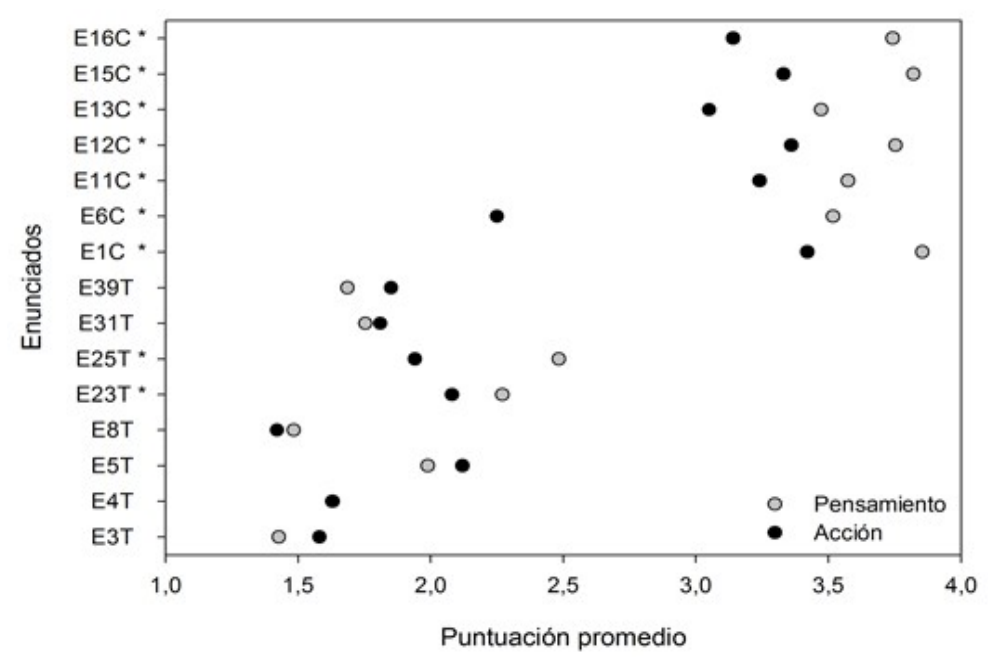

Figura 2. Relación entre lo declarado "Pensamiento" y "Acción" para cada uno enunciado (* indica diferencias significativas, $\mathrm{p}<0,05)$.

La "ruptura" de la que hablamos entre Pensamiento y Acción también se aprecia para enunciados de tendencia tradicional (E23T y E25T) en ello advertimos que usar evaluaciones sumativas para mantener el interés de los estudiantes o para medir el aprendizaje de conceptos científicos, son asuntos que el profesor no recomienda en la planificación, sin embargo, las condiciones laborales contextuales que enfrenta, por ejemplo, calificar a los estudiantes en períodos breves de tiempo, llevan al profesor a implementar una evaluación tradicional centrada en el contenido disciplinar.

\section{b) Creencias del profesor de biología y la evaluación docente en Chile}

En Chile la Evaluación Docente busca promover la calidad de la educación a través del fortalecimiento de la profesión docente (Tejeda 2011). Nuestro análisis se focaliza en las dimensiones de desempeño: Organización de Elementos de la Unidad (OEU), Evaluación de los Aprendizajes (EA) y Reflexión Pedagógica (RP), los que en los últimos 4 años (Figura 3) presentan un alto porcentaje de profesores en los niveles Insatisfactorio -profesor que presenta debilidades en el indicador- y Básico -profesor que cumple con lo esperado en el indicador, pero ocasionalmente- (Flotts y Abarzúa 2011). Cabe señalar que los resultados de la 
última evaluación docente publicada (2016) no tiene disponible los resultados para las dimensiones que se han considerado en este artículo.

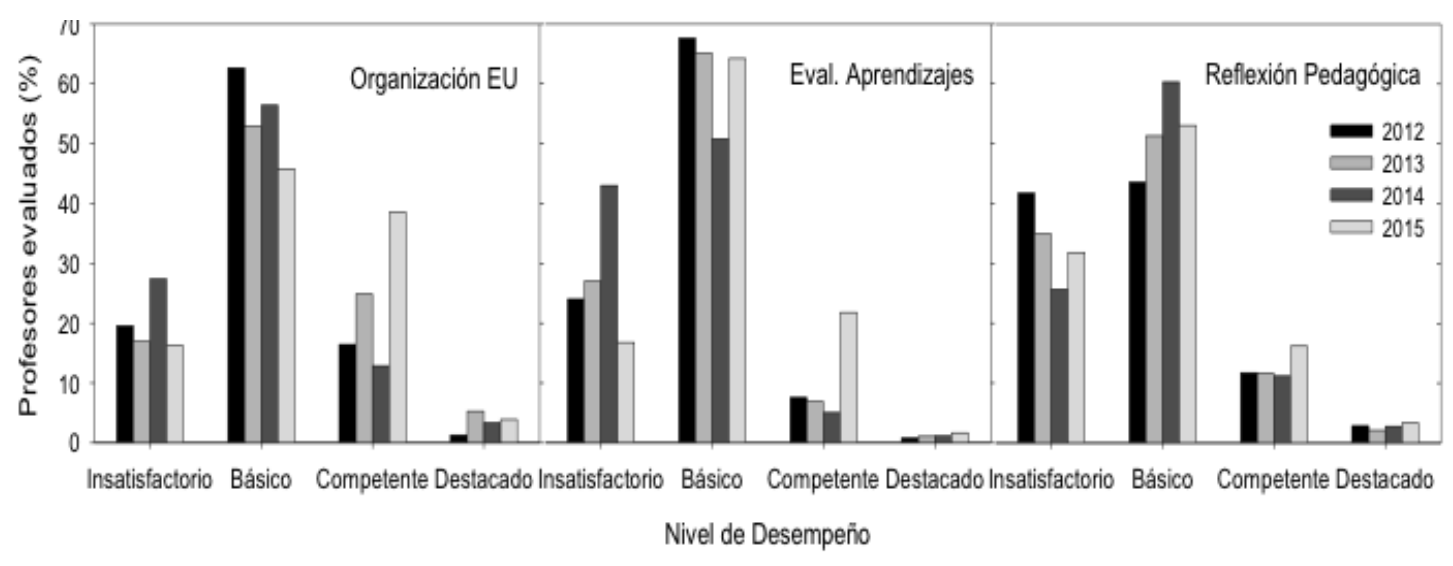

Figura 3. Resultados para los indicadores a) Organización de Elementos de la Unidad, b) Evaluación de los Aprendizajes y, c) Reflexión Pedagógica de la EDD (2012-2015).

b1) Organización de los Elementos de la Unidad

La EDD en el período de 2012 a 2015 da cuenta que menos el 50\% de los profesores evaluados en el período no alcanzan un nivel de desempeño adecuado en la dimensión (profesor Competente) o un desempeño que esté por sobre lo esperado (profesor Destacado) en acciones como: Secuencia de clase, contribución de las actividades al logro de los objetivos o capacidad para reformular las estrategias didácticas, siendo estas acciones a considerar en la PEB (Tabla 3), no obstante, su implementación es difícil, especialmente aquellas vinculadas con la reformulación de estrategias didácticas (E12C) o el diseño de actividades para socializar un objetivo de aprendizaje (E11C). Sobre lo último, entre el año 2015 y 2016 con 5 profesores de biología en servicio identificamos dos razones de por qué no socializar los objetivos usando una secuencia de actividades, que son: 1) temor de invertir demasiado tiempo en su implantación descuidando la enseñanza de los contenidos conceptuales de biología prescritos en el currículo; 2) no saber diseñar una secuencia de actividades que permita la comprensión y sentido de los objetivos de aprendizaje en la clase, representando esta situación una oportunidad de trabajo para el desarrollo del profesor.

b2) Evaluación del aprendizaje

Algunos piensan que el análisis de las creencias sobre la evaluación de los aprendizajes tiene sentido para entender la relación entre las creencias y los resultados de aprendizaje, dado que un cambio de creencia sobre la evaluación modificaría los métodos de evaluación (Opre 2015), y, por consiguiente, los resultados del aprendizaje. Siendo la evaluación del aprendizaje unos de las dimensiones de desempeño profesional docente, su exploración es importante, especialmente cuando en el período 2012-2015, menos del 20\% de los profesores evaluados alcanzan un desempeño adecuado o por sobre lo exigido. En un plano declarativo, los profesores comparten una noción de evaluación propia del paradigma de autorregulación, en la que la evaluación sumativa no debe ser usada como una forma para mantener el interés (E23) o medir el aprendizaje de conceptos biológicos de los estudiantes (E25); sin embargo, se cree promover una evaluación contraria a lo que se piensa (Figura 2), antecedentes que coinciden con lo señalado por Davis y Neitzel (2011) quienes señalan que el profesor no tiende a usar métodos formativos de evaluación. 


\section{b3) Reflexión Pedagógica}

Esta dimensión representa para la política pública chilena la capacidad del profesor para remirar los aspectos de su práctica de enseñanza, cuestión que exige además, una actitud crítica, particular y permanente, que busque transformarse en un hábito deliberado y no en una acción aislada condicionada por las exigencias y consecuencias de la EDD. En Chile, los resultados de la EDD en el periodo 2012-2015 no han sido del todo alentadores, ya que aún es bajo el porcentaje de los profesores en el nivel de desempeño Competente $(X=12.7 \%$ ) o Destacado $(\mathrm{X}=2.8 \%)$ en lo que es reflexión pedagógica, a pesar de declarar que el análisis de resultados de enseñanza y aprendizaje (E15C, E13C) constituye una cuestión que se hace con frecuencia cuando prepara la enseñanza de la biología.

\section{Conclusiones e implicaciones para el desarrollo profesional del profesor}

El estudio nos permite concluir que todas aquellas ideas que extrapolan, en un plano declarativo, decisiones o acciones docentes de tendencia constructivista dan sentido a las decisiones que toma el profesor y por ello, muestran una alta adhesión, exceptuando lo referido al diseño de estrategias de evaluación entre pares (E6C) vista más bien como una acción solitaria, para lo cual, al parecer, no existe costumbre de socializar dichas estrategias entre pares.

En el plano del conocimiento de la materia, el profesor adscribe a la necesidad de vincular los contenidos científicos unos con otro (E1C), aunque el 89,2\% de ellos piensa que lo adecuado es organizarlos en una secuencia lógica y lineal (E4T) que responda a la lógica de la disciplina $(\mathrm{E} 31 \mathrm{~T})$. Estas creencias, indican que la organización de la materia se representa como un listado de conceptos científicos para el profesor y como un vocabulario específico para el estudiante (Astolfi 1997), descuidando su valor como estrategia de pensamiento y transformador de ideas. El conocimiento de la materia no solo se remite a los hechos, teorías y principios, sino también a las maneras de cómo la disciplina evalúa y acepta el nuevo conocimiento (Schwab 1978, Grossman et al. 2005), en ese sentido, es importante que el profesor comprenda ampliamente la materia que enseña para una enseñanza eficaz, para lo cual debe evitar enseñar los conceptos científicos como enunciados únicos, sin considerar su estructura de organización interna (Astolfi 1997), cuestión que el profesor con frecuencia cree no hacer, aunque en este estudio evidenciamos lo contrario.

El profesor de biología cree que la evaluación del aprendizaje se planifica -se dice hacer- para medir el aprendizaje de conceptos científicos (E25T; 79.5\%), aun cuando no es fundamental considerarla en la planificación de la enseñanza de la biología (E25T; 45.8\%). Dicha situación deja entrever que la evaluación no pretende, en este sentido, ser una estrategia para ayudar a otros a transformar las ideas o ampliar su comprensión sobre lo discutido, ya que son evaluaciones que se centran en los conceptos científicos, lo que no favorece la comprensión de fenómenos o ideas de ciencia (Harlen 2010). Esto constituye una controversia desde el punto de vista del profesor y sus creencias, ya que adopta la idea de vincular los contenidos científicos para ampliar la comprensión de los estudiantes, en consecuencia, la evaluación se reduce al concepto científico.

Evidenciamos que la coherencia entre lo que se piensa se debe hacer y lo que se piensa que se hace es significativamente diferente (Figura 2) cuando los enunciados aluden a una creencia de tipo constructivista, reflejando que los significados del profesor valoran estos enfoques, aun cuando la acción está representada por un enfoque tradicional. Desde el punto de vista del pensamiento, -lo que se cree se debe hacer- sobre la Organización de los Elementos de la Unidad (OEU) y Reflexión Pedagógica (RP) movilizan al profesor, especialmente, por develar 
una noción de aprendizaje como proceso gradual y de atención, donde los objetivos, las actividades y la evaluación del aprendizaje responden a las dificultades de aprendizaje de los estudiantes, siempre que los productos del análisis sean considerados en la preparación de la enseñanza como dicen que piensan y hacen los profesores encuestados (E15C). Así, la secuencia de clases (Tabla 3) y el análisis de decisiones pedagógicas (Tabla 4), en particular lo referido a los aprendizajes deseados, representa una serie de creencias ingenuas influenciadas por el currículum prescrito, siendo la más reveladora aquella que sostiene que los contenidos de biología se deben organizar -y los organizo- en una secuencia lineal que se ajuste a la lógica de la disciplina que enseño (E31T).

Por cierto, la reflexión sobre la práctica de enseñanza debe permitir la construcción de conocimiento profesional y la valoración de las decisiones pedagógicas y el pensamiento del profesor (Loughran, Keast y Cooper 2016) en y para una buena enseñanza (Fenstermacher 1989), particularmente cuando se ha evidenciado falta de teorización (Bravo y Cofré 2016) y de reconocer que la relación de la teoría y la práctica influyen en la enseñanza (Loughran y Hamilton, 2016) y su preparación, representando en ese sentido, un foco importante de desarrollo profesional. En otro marco, la exploración de las creencias y el desempeño del profesor deben ayudarnos a generar bases de orientación profesional, sustentadas en una reflexión activa y consciente, sobre todos aquellos asuntos que resulten una preocupación, una dificultad o un problema de enseñanza para el profesor (Ravanal 2017), reconociendo la naturaleza compleja y problemática de la preparación y enseñanza de la biología y la necesidad de re-conceptualizarla para comprenderla desde todas aquellas representaciones que median las decisiones y acción del profesor de biología.

\section{Agradecimientos}

Programa Conicyt- Fondecyt de iniciación No11140297 del primer autor (ERM). A programa de Doctorado de la Universidad Academia de Humanismos Cristiano (FLP). A programa de Doctorado de la Universidad Metropolitana de Ciencias de la Educación (LRM).

\section{Referencias}

Astolfi J. (1997) Conceptos clave en la didáctica de las disciplinas. Sevilla: Díada.

Bahçivan E. (2016) Investigating the relationships among PSTs' teaching belief: are epistemological belief central? Educational Studies 42 (2), 221-238.

Batatia H., Hakkarainen K., Mørch A. (2012) Tacit knowledge and trialogical learning: Towards a conceptual framework for designing innovative tools. En A, Morch \& S, Paavola, Collaborative knowledge creation (pp. 15-30). Rotterdam, The Netherlands: Sense Publisher.

Berlanga V., Rubio M.J. (2012) Clasificación de pruebas no paramétricas. Cómo aplicarlas en SPSS. Revista dinnovació i recerca en Educació 5(2), 101-113.

Bravo P., Cofré H. (2016) Developing biology teachers pedagogical content knowledge through learning study: the case of teaching human evolution, International Journal of Science Education 38(16), 2500-2527.

Bryan L. (2012) Research on science teacher belief. En B, Fraser, K, Tobin \& C, McRobbie (Eds), Second International Handbook of Science Education (pp. 477- 498). New York: Springer International. 
Buehl M., Beck J. (2015) The relationship between teachers' belief and teacher' practices. En H, Fives \& M.G, Gill (Eds.), International Handbook of Research on Teachers'Belief (pp. 6684). New York: Routledge.

Contreras, S.A. (2010) Las creencias y actuaciones curriculares de los profesores de ciencias de secundaria de Chile. (Tesis doctorado, Universidad Complutense de Madrid). Recuperada de http://eprints.ucm.es/11624/1/T32362.pdf

Davis D., Neitzel C. (2011) A self-regulated learning perspective on middle grades classroom assessment. Journal of Educational Research 104 (3), 202-215.

Fenstermacher G. (1989) Tres aspectos de la filosofía de la investigación sobre la enseñanza. En M. Wittrock. La investigación de la enseñanza I Enfoques, teorías y métodos (pp. 150-181). Paidós: Madrid.

Fives H., Buehl M. (2012) Spring cleaning for the "messy" construct of teachers' beliefs: What are they? Which have been examining? What can they tell us? En K, Harris \& T, Urdan (Eds.), APA Educational Psychology Handbook Vol. 2 Individual differences and cultural and contextual factors (pp. 471-499). Washington, DC: US.

Flotts M., Abarzúa A. (2011) El modelo de evaluación y los instrumentos. En J, Manzi, R, González \& Y, Sun (Eds.), La evaluación docente en Chile (35-62). Santiago: MIDE UC, Pontificia Universidad Católica de Chile.

Grangeat M, Kapelari, S. (2015) Introduction: Exploring the growth of science teacher`s professional knowledge. En M. Grangeat (Ed.), Understanding science teacher's professal knowledge growth (pp. 1-12). Rotterdam, The Netherlands: Sense Publishers.

Grossman P., Wilson S., Shulman L. (2005) Un estudio comparado: las fuentes del conocimiento didáctico del contenido en la enseñanza del inglés en Secundaria. Revista de Curriculum y Formación del Profesorado 9 (2), 1-18.

Guerra P., Sebastián, C. (2015) Creencias epistemológicas en profesores que postulan a programas de Acreditación de Excelencia Pedagógica. Análisis descriptivos y comparativos entre profesores que se desempeñan en los diferentes niveles de enseñanza. Revista Estudios Pedagógicos XLI (2), 107-125.

Harlen W. (2010) Principles and big ideas of science education. Hatfield, Herts: Association for Science Education.

Hofer B., Pintrich P. (1997) The Development of Epistemological Theories. Review of Educational Research 67, 88-140.

Hoffman B., Seidel K. (2015) Measuring teachers' belief. En H, Fives \& M.G, Gill (Eds.), International Handbook of Research on Teachers'Belief (pp. 106-127). New York: Routledge.

Hutner T., Markman A. (2016) Proposing an operational definition of science teacher belief. Journal Science Teacher Educational 27 (6), 675-691.

Kang N.H. (2008) Learning to teach science: Personal epistemologies, teaching goals, and practice of teaching. Teaching and Teacher Education 24 (2), 478 - 498.

Kennedy M. (1998) Education Reform and subject matter knowledge. Journal of Research in Science Teaching 35 (3), 249-263.

Kind V. (2015) On the beauty of knowing then not knowing. En A. Berry, P. Friedrichsen \& J. Loughran (Eds.), Re-examining Pedagogical Content Knowledge in Science Education (pp. 178195). New York: Routledge. 
Lawshed C. (1975) A quantitative approach to content validity. Personal Psychology, 28 (4), 563 575.

Lee M., Tsai C. (2011) Teachers'scientific epistemological views, conceptions of teaching science and their approaches to teaching science: An exploratory study of in-service science teachers in Taiwan. En J, Brownlee, G, Schraw \& D, Berthelsen (Eds.), Personal epistemology and teacher education (pp. 246-262). London: Touteledge.

Loughran J., Keast S., Cooper R. (2016). Pedagogical reasoning in teacher education. En Loughran, J. \& Hamilton, M. (Eds.), International Handbook of Teacher Education (pp. 387421). Singapore: Springer.

Loughran J., Hamilton M. (2016) Developing an understanding of teacher education. En Loughran, J. \& Hamilton, M. (Eds.), International Handbook of Teacher Education (pp. 322). Singapore: Springer.

Mansour N., Wegerif R. (2013) Why science educatoon for diversity? En N, Mansour \& R. Wegerif (Eds.), Science Education for Diversity. Theory and Practice (pp. ix-xx). Cultural Studies os Science Education: Springer.

Ministerio de Educación del Gobierno de Chile (2008). Marco para la Buena Enseñanza. Santiago: Centro de Perfeccionamiento, Experimentación e Investigación Pedagógica.

Opre D. (2015) Teachers' conceptions of assessment. Procedia-Social and Behavioral Sciences 209, 229-233.

Park S., Suh J., Seo K. (2017) Devolopment and validation of measures of secondary science teachers'PCK for teaching photosynthesis. Research in Science Education, 1-25 doi: 10.1007/s11165-016-9578-y.

Pujalte A., Adúriz-Bravo A., Porro S. (2015) Las imágenes de la ciencia en profesoras y profesores de biología: entre lo que se dice y lo que se hace. Revista Boletín Biológica 3, 510.

Quintanilla M. (2006) La ciencia en la escuela: un saber fascinante para aprender a `leer el mundo'. Pensamiento Educativo 39 (2), 177-204.

Ravanal E., Quintanilla M. (2012) Concepciones del profesorado de biología en ejercicio sobre el aprendizaje científico escolar. Enseñanza de las Ciencias 30 (2), 33-54.

Ravanal, E. (2017) Consideraciones para un programa de desarrollo profesional que orienta al profesor a re-conceptualizar su enseñanza. Revista Científica 28, 60-71.

Rollnick M., Mavhunga E. (2015) The PCK Summit and its effect on work in South Africa. En A. Berry, P. Friedrichsen \& J. Loughran (Eds), Re-examining Pedagogical Content Knowledge in Science Education (pp. 135-146). New York: Routledge.

Scheerens J. (2016) Educational effectiveness and ineffectiveness. Dordrecht: Springer.

Schwab J. (1978) Science, curriculum and liberal education. Chicago, IL: Universidad de Chicago Press.

Soini T., Pietarinen J., Pyhältö K. (2016) Leading a school through change - principals' handson leadership strategies in school reform. School Leadership \& Management 36 (4): $452-$ 469.

Tejeda J. (2011) Evaluación del desarrollo profesional docente basado en competencias. En C, Marcelo (Coord.), Evaluación del desarrollo profesional docente (pp. 23-47). Barcelona: Díada. 
Torres J., Perera V. (2009) Cálculo de la fiabilidad y concordancia entre codificadores de un sistema de categorías para el estudio del foro online en E-Learning. Revista de Investigación Educativa 27(1), 89-103.

Tristán-López A. (2008) Modificación al modelo de Lawshe para el dictamen cuantitativo de validez de contenido de un instrumento objetivo. Avances en Medicina 6, 37-48.

Wallace J., Loughran J. (2012) Science teacher learning. En Fraser, B., Tobin, K. \& McRobbie, C. (Eds.), Second International Handbook of Science Education (pp. 295-306). New York: Springer.

Wallace C. (2015) Overwiew of the role of teacher belief in science education. En R, Evans, J, Luf, C, Czerniak, \& C, Pea (Eds.), The role of science teachers' belief in international classroom (pp. 17-34). Rotterdam The Netherlands: Sense Publishers. 